\title{
Identification of effective properties of particle reinforced composite materials
}

\author{
V. Kushnevsky, 0. Morachkovsky, H. Altenbach
}

\begin{abstract}
For the determination of effective elastic properties an energy averaging procedure has been used for particle reinforced composite materials. This procedure is based on finite element calculations of the deformation energy of a characteristic volume element. The proposed approach allows the determination of effective properties of particle reinforced composite with acceptable precision. The calculated effective properties of the composite are found in range between upper and lower Hashin-Shtrikman bounds. The averaging elastic properties of the composite depend on the properties of the particles, matrix volume fraction of the particles and some parameters taking into account the influence of the interphase between matrix and particles. These dependencies can be presented by simple analytical functions approximatically. An identification procedure basing on numerical experiments allows the estimation of the unknown approximation parameters. The obtained functions describe precisely the numerical data for any relationship between material constituents.
\end{abstract}

\section{1}

\section{Introduction}

The wide use of reinforced materials in constructions in the last years initiate the development of new techniques for measuring the material's properties, because the classical techniques did no longer satisfy the needs (Sol, 1993). The heterogeneous character of these materials at the microscopic level leads to difficulties in mechanical modelling. Developments in computer science and the availability of

Communicated by S. N. Atluri, 28 April 1998

V. Kushnevsky ${ }^{1}$, O. Morachkovsky², H. Altenbach

Institut für Werkstoffwissenschaften,

Martin-Luther-Universität Halle-Wittenberg,

D-06099 Halle, Germany

Present addresses:

${ }^{1}$ Institute of Computer Analysis of Structures,

Riga Technical University, LV- 1658 Riga Latvia

${ }^{2}$ Visiting professor, on leave from chair of Theoretical Mechanics,

Kharkov State Polytechnical University, ul. Frunze,

21 UKR- 310002 Kharkov, Ukraine

Correspondence to: $\mathrm{H}$. Altenbach

The visit of the second author was financially supported by the Martin-Luther-Universität Halle-Wittenberg. numerical techniques for solving problems in solid Emechanics has allowed to overcome a part of these difficulties. All above have made it possible to develop a new hybrid technique for material identification, the so-called mixed numerical-experimental technique (Sol, 1986).

The mixed numerical-experimental method connects experimental data with calculated data. It allows to obtain properties of the material that was described analytically and then refined by experimental data. The numerical model can be made more precise, when calculated and measured data are nearly the same. Sol (1986) applied this procedure to anisotropic plates, where the varying parameters are the elastic material constants, and the measured and calculated data are the resonance frequencies of the free plate.

While the numerical model is based on different hypotheses, the mixed numerical-experimental methods lead to modeling and numerical errors, because the real structure do not satisfy one or more of these hypotheses and the structures is discretisized approximately. The aim of the development of mixed numerical-experimental techniques is to obtain a method that minimize the model errors (Sol, 1986; Bolognini, Rccio and Bettianli, 1993; Mota Soares, Morelira De Freitas, Araujo and Pedersen, 1993).

Recently many different approaches are used for the identification of the physical parameters directly characterizing structural behaviour (e.g. Young's modulus and density of the material). Bolognini et al. (1993) use the appropriate comparison between actual eigenpairs of an existing structure and eigenpairs obtained by finite element analysis. It leads to the identification of parameters, which can be used for the calibrating of the model as well as for the detection of damaged zones in the structure. Numerical experimental identification methods are used mainly in structural applications. The determination of stiffness parameters for complex materials such as fibre reinforced composites is much more complicated than for isotropic materials. This is not only caused by the high number of independent parameters but also because the anisotropy of these materials raises specific problems, when traditional tests are employed in an attempt to evaluate the desired quantities in a direct way. One of the approaches to determine the effective properties of the composite is their identification from vibration tests. This method was first developed for the thin plates modeled by the classical plate theory (Pedersen, 1990; Pedersen and Frederiksen, 1992). Later, the method was extended to the case of thick plates. The use of such plates in conjuction with frequencies of higher modes assures a significant 
transverse shear effect. This enables the identification of the two transverse shear moduli. Preliminary papers that consider the identification of thick plates were presented by Frederiksen (1992) and Frederiksen and Pedersen (1993). The numerical model used for the solution of the vibration problem of thick plates with free edges comprises a Ritz formulation of a higher-order plate theory. The theoretical aspects of the recent estimation procedure including numerical simulation are described by Frederiksen (1997a). A description of the experimental procedure and practical examples are given in (Frederikesen, 1997b). A different approach for the identification of thick plate constants is based on the finite element formulation using a higher order plate theory. Mota Soares et al. (1993) developed this model for the identification of thick plates with the assumption of transverse isotropy. Later, Araujo, Mota Soares and Moreira De Freitas (1996) expanded this approach to determine six constants of a thick plate. The identification results of both approaches have been compared and have shown a good correspondence.

The goal of the present investigation is to apply the first part (numerical identification) of the mixed numerical-experimental technique for the determination of the average properties of the particle reinforced composite materials.

\section{2}

\section{Averaging procedures}

The dual character of the composite material (at the macrolevel the composite materials are homogeneous, at the microlevel they are heterogeneous) requires to introduce different models of the micromechanical behaviour of the composite. Averaged properties of the composite, obtained by means of micromechanical models, allow to analyse the stress behaviour of the structural elements. Different approaches have been proposed to determine the effective or averaged properties for composites from known properties of its constituents (Rammerstorfer and Böhm, 1994). First analytical investigations of mechanical properties of composite materials have been described in a paper by Einstein (1906). He has determined the effective viscosity of a fluid containing a small amount of rigid spherical particles. Until about 1960, investigations have been primarily concerned with macroscopically isotropic composites. While in 60ies composites became widely used as construction materials, investigations of the effective mechanical properties of composite materials were started. The most significant research was performed by Eshelby (1957) that established the so-called Direct Approach. Methods based on this approach have been applied only for models with particles of relative simple geometrical shapes. Due to mathematical difficulties, the effective elastic properties of the composite are calculated by an exact solution of a boundary value problem for a certain geometrical model of the material. Eshelby considered the simplest case - a dilute concentration of spherical or ellipsoidal particles of material 2 in matrix 1 . The definition of "dilute" means that the state of strain in any particle in the composite body under homogeneous boundary conditions is not affected by the other particles. Therefore the strain is that of a single particle in an infinite body and this happens to be uniform for an ellipsoid with far field ho- mogeneous strain. For spherical particles the effective bulk and shear moduli of a composite can be calculated :

$$
\begin{aligned}
K_{\mathrm{c}}=K_{\mathrm{m}}+ & \left(K_{\mathrm{p}}-K_{\mathrm{m}}\right) \frac{3 K_{\mathrm{m}}+4 G_{\mathrm{m}}}{3 K_{\mathrm{p}}+4 G_{\mathrm{p}}} \phi \\
G_{\mathrm{c}}=G_{\mathrm{m}}+ & \left(G_{\mathrm{p}}-G_{\mathrm{m}}\right) \\
& \times \frac{5\left(3 K_{\mathrm{m}}+4 G_{\mathrm{m}}\right)}{9 K_{\mathrm{m}}+8 G_{\mathrm{m}}+6\left(K_{\mathrm{m}}+2 G_{\mathrm{m}}\right) G_{\mathrm{p}} / G_{\mathrm{m}}} \phi
\end{aligned}
$$

Here, subscript $c, m$ and $p$ refer to the composite, matrix and particles, respectively. $\phi$ is the volume fraction of the particles $(\phi \ll 1), K, G$ - bulk and shear moduli.

This work has been the base for most future investigations. Eshelby's Direct Approach has been extended by Hashin (1962) to Composite Sphere Model, that allows to calculate composites with different spherical particles sizes. The effective bulk modulus for this model is

$K_{\mathrm{c}}=K_{\mathrm{m}}+\left(K_{\mathrm{p}}-K_{\mathrm{m}}\right) \frac{\left(3 K_{\mathrm{m}}+4 G_{\mathrm{m}}\right) \phi}{3 K_{\mathrm{p}}+4 G_{\mathrm{m}}+3(1-\phi)\left(K_{\mathrm{p}}-K_{\mathrm{m}}\right)}$

For these models the exact solution of the effective shear modulus was not obtained. It was the reason to develop the Variational Bounding Approach (Hashin and Shtrikman, 1962). Variational Bounding Approaches are useful for the calculation of upper and lower bounds of composite's elastic constants composites. Lower and upper bounds are then obtained by minimizing the complementary energy or the potential energy, respectively. Bounds for two-phase materials have been determined by Hashin and Shtrikman $(1962,1963)$. For isotropic phases, the bounds for bulk and shear moduli can be written as (index $\mathrm{u}$ - upper bound, index 1 - lower bound)

$$
\begin{aligned}
& K_{\mathrm{c}}^{\mathrm{u}}=K_{\mathrm{p}}+\frac{\left(K_{\mathrm{m}}-K_{\mathrm{p}}\right)\left(3 K_{\mathrm{p}}+4 G_{\mathrm{p}}\right)(1-\phi)}{\left(3 K_{\mathrm{p}}+4 G_{\mathrm{p}}\right)+3\left(K_{\mathrm{m}}-K_{\mathrm{p}}\right) \phi} \\
& K_{\mathrm{c}}^{\mathrm{l}}=K_{\mathrm{m}}+\frac{\left(K_{\mathrm{p}}-K_{\mathrm{m}}\right)\left(3 K_{\mathrm{m}}+4 G_{\mathrm{m}}\right) \phi}{\left(3 K_{\mathrm{m}}+4 G_{\mathrm{m}}\right)+3\left(K_{\mathrm{p}}-K_{\mathrm{m}}\right)(1-\phi)} \\
& G_{\mathrm{c}}^{\mathrm{u}}=G_{\mathrm{p}}+\frac{5 G_{\mathrm{p}}\left(G_{\mathrm{m}}-G_{\mathrm{p}}\right)\left(3 K_{\mathrm{p}}+4 G_{\mathrm{p}}\right)(1-\phi)}{5 G_{\mathrm{p}}\left(3 K_{\mathrm{p}}+4 G_{\mathrm{p}}\right)+6\left(G_{\mathrm{m}}-G_{\mathrm{p}}\right)\left(K_{\mathrm{p}}+2 G_{\mathrm{p}}\right) \phi}
\end{aligned}
$$

$$
\begin{aligned}
& G_{\mathrm{c}}^{\mathrm{l}}=G_{\mathrm{m}} \\
& +\frac{5 G_{\mathrm{m}}\left(G_{\mathrm{p}}-G_{\mathrm{m}}\right)\left(3 K_{\mathrm{m}}+4 G_{\mathrm{m}}\right) \phi}{5 G_{\mathrm{m}}\left(3 K_{\mathrm{m}}+4 G_{\mathrm{m}}\right)+6\left(G_{\mathrm{p}}-G_{\mathrm{m}}\right)\left(K_{\mathrm{m}}+2 G_{\mathrm{m}}\right)(1-\phi)}
\end{aligned}
$$

where $K_{\mathrm{p}}>K_{\mathrm{m}}$ and $G_{\mathrm{p}}>G_{\mathrm{m}}$.

Improved bounds can be calculated considering information about the phase geometry. Using the admissible fields obtained from a sheared composite spheres the following upper bound for $G$ can be determined (Hashin, 1983)

$$
\begin{aligned}
& G_{\mathrm{c}}^{\mathrm{u}}=G_{\mathrm{m}} \\
& +\left[1+\frac{\phi}{1 /(\gamma-1)+A(1-\phi)-\phi\left(1-\phi^{2 / 3}\right)^{2} /\left(B \phi^{7 / 2}+C\right)}\right]
\end{aligned}
$$


Here, the variables $\gamma, A, B$ and $C$ (Hashin, 1983) depend on the elastic properties of the phase components. The lower bound remains as in equation (7).

Other branch of investigations based on the Eshelby solution, the Approximation Method, is mostly derived for models with specified phase geometries. The best-known Approximation Method for particle composites is the selfconsistent method (SCM) proposed independently by Budiansky (1965) and Hill (1965). A typical ellipsoidal or spherical inclusion is embedded in a homogeneous, uniformly stressed or strained infinite body which has the unknown properties $K_{\mathrm{c}}$ and $G_{\mathrm{c}}$. The properties $K_{\mathrm{c}}$ and $G_{\mathrm{c}}$ are calculated using Eshelby's solution (Eshelby, 1957).

A more realistic version of SCM is known as the generalized self consistent method (GSCM). In this method, the spherical particle is embedded in a concentric spherical shell of the matrix material. Shell and particle dimensions are chosen to correspond to the prescribed volume fraction, and the particle-shell assembly is embedded in an infinite medium with unknown effective properties $K_{\mathrm{c}}$ and $G_{\mathrm{c}}$. The solution for $G_{\mathrm{c}}$ is the solution of a quadratic equation

$A\left(G_{\mathrm{c}} / G_{\mathrm{m}}\right)+2 B\left(G_{\mathrm{c}} / G_{\mathrm{m}}\right)+C=0$,

where $A, B$ and $C$ are defined by Christensen and Lo (1979). For the bulk modulus Christensen treated the same result as Hashin (1962).

Roscoe (1952) developed and applied a Differential method to model the behaviour of fluid suspensions. The composite material can be regarded as the result of a sequence of incremental additions of filler particles to the matrix material (Mc Laughlin, 1977; Norris, 1985; Christensen, 1990). The determination process starts with the pure matrix phase. Filler grains are added incrementally, that their concentration increases from zero to the final value. At each stage, the added particles are assumed to be embedded in a homogeneous material composed of a pure matrix and previously added filler grains.

Composite materials are used to fabricate large structural components. However, the behaviour of these structures depends on the composite microstructure. Analysing large structureson a microlevel seems impossible. Analytical methods have therefore sought to approximate composite structural mechanics by analysing a representative section of the composite microstructure, commonly called Representative Volume Element (RVE) (Hill, 1965;
Hashin, 1983; Sun, Wang and Yu, 1991; Hollister and Kikuchi, 1992; Chingshen and Ellyin, 1994; Wu, Meng and Du, 1997).

RVE based methods divide the analysis of a composite material into analyses at the local and global levels. On the local microstructural level effective properties of the RVE are determined. The composite structure is then replaced by an equivalent homogeneous material having the calculated effective properties. The global level analysis calculates the effective stress and strain within the equivalent homogeneous structure. The process of calculating effective properties has been termed "homogenization" by Suqet (1987).

A numerical approach for the identification of effective properties of a particle reinforced composite material is developed in this article. Random distribution of particles in structure is approximated by a periodic array which is similar to a cubic lattice, as shown in Fig. 1. The size of each particle is equal to the average particle size of the composite. Such RVE of the composite material is analysed with the finite element method (ANSYS ${ }^{\odot} 5.3,1996$ ). Uniform boundary displacements or tractions are applied at the boundaries of the RVE to introduce either a known average strain or average stress in the RVE. Local stress and strain distributions in the RVE are calculated using the finite element method. Different effective elastic constants can be calculated by averaging local fields corresponding to different sets of boundary conditions.

The basic assumptions of the presented model are the following: the particles have spherical form; both matrix and particles are homogeneous, isotropic and linear elastic; adjacent constituents (phases) are perfectly bonded.

It is assumed that the region of interest does have a sufficient distance from the points of a load application or geometric constraints. Each particle and its surrounding matrix in that region will gain the same deformation under applied loads, therefore only a single particle and its surrounding matrix have to be analysed. Thus, the properties of the composite may be studied considering only a small region or a representative volume element.

\section{3 \\ Finite element approach}

Since above proposed approximation of the composite structure as cubic lattice, the composite structure consists of rectangular volumes (superelements) which repeat

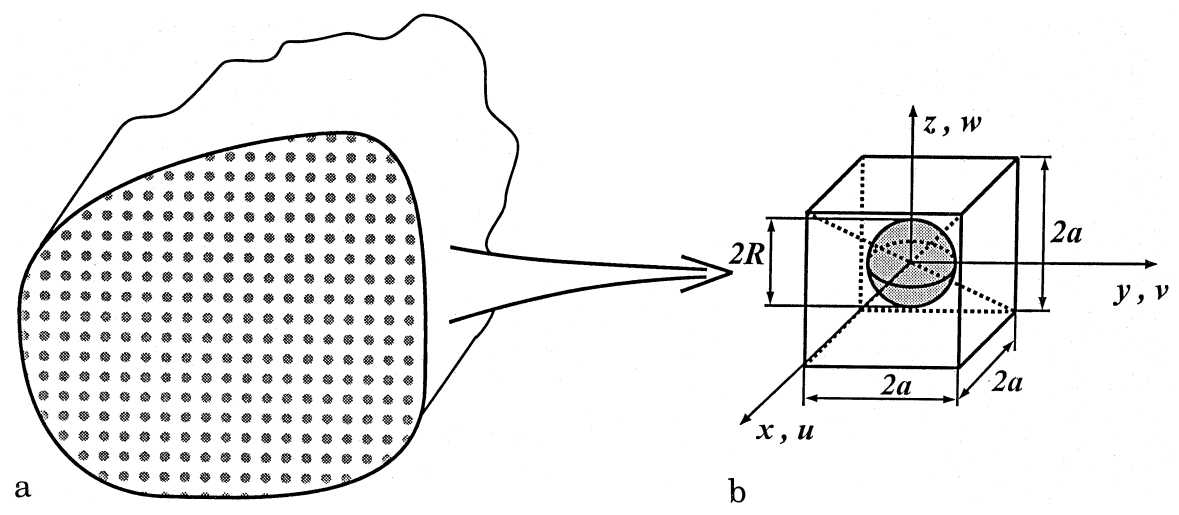

Fig. 1. a, b Particle reinforced composite; Representative volume element 
themselves periodically (Fig. 1). This representative volume element is chosen as a basic cell of the composite medium. The equivalent material which is generally an anisotropic homogeneous medium is represented by the same volume and exhibits the same strain energy in the deformed state as the real composite. Thus,

$U=U^{\mathrm{eq}}, \quad V=V^{\mathrm{eq}}$,

where $V$ and $U$ are volume and strain energy of the real composite, $V^{\text {eq }}$ and $U^{\text {eq }}$ are the same quantities for the equivalent material, respectively. Such approach was used by Rikards, Krasnikov and Kushnevsky (1991) for the determination of elastic characteristics of composite materials.

Let us denote the macrostrain of a superelement by $E_{i j}$ and the corresponding macrostresses by $S_{i j}$. Then, the strain energy of the superelement treated as an equivalent material (macrohomogeneous body) can be expressed as

$U^{\mathrm{eq}}=\frac{1}{2} \int_{V} S_{i j} E_{i j} \mathrm{~d} V=\frac{1}{2} \int_{V} C_{i j k l} E_{i j} E_{k l} \mathrm{~d} V$,

where $C_{i j k l}$ are the stiffness tensor components of the superelement treated as macrohomogeneous body. On the other hand, the strain energy of the superelement as a composite treated as nonhomogeneous medium has been computed using the finite element method. In this case, the corresponding strain energy of the superelement can be derived by the relationship

$U=\frac{1}{2} \int_{V} \sigma_{i j} \varepsilon_{i j} \mathrm{~d} V$

where $\sigma_{i j}$ and $\varepsilon_{i j}$ are microstresses and microstrains in the superelement.

Using Eq. (10) and taking into account Eq. (9) one obtains

$\frac{1}{2} \int_{V} C_{i j k l} E_{i j} E_{k l} \mathrm{~d} V=\frac{1}{2} \int_{V} \sigma_{i j} \varepsilon_{i j} \mathrm{~d} V$,

This equation provides a linear relationship for the determination of stiffness matrix components $C_{i j k l}$ of the equivalent material. Apparently one has to introduce a sufficient number of admissible problems related to the superelement to get the necessary linear algebraic equations which provide the desired elastic constants. The unidirectional particle reinforced composite (see Fig. 1.) is considered as macroisotropic homogeneous material

$$
\begin{aligned}
& C_{1111}=C_{2222}=C_{3333}, \\
& C_{1122}=C_{2233}=C_{1133}, \\
& C_{2323}=C_{1212}=C_{1313}=\frac{1}{2}\left(C_{1111}-C_{1122}\right),
\end{aligned}
$$

with two independent elasticity components $C_{1111}$ and $C_{1122}$. All other $C_{i j k l}$ are zero. For the determination of these constants, two deformation states of the superelement with following boundary conditions are considered:

$$
\begin{array}{rrrr}
\text { I } & v(x, y, z)=u & w(x, y, a)=0 \\
u(-a, y, z)=-u & v(x,-a, z)=0 & w(x, y,-a)=0 \\
\text { II } \quad u(a, y, z)=0 & v(x, a, z)=v & w(x, y, a)=0 \\
u(-a, y, z) & =0 & v(x,-a, z)=-v & w(x, y,-a)=0
\end{array}
$$

The two boundary problems (13) are solved to determine the effective elastic constants (12) using the equilibrium (11)

$$
\begin{aligned}
& \frac{1}{2} C_{1111}\left(E_{11}^{I}\right)^{2}=U_{I} \\
& \frac{1}{2} C_{2222}\left(E_{22}^{I I}\right)^{2}=U_{I I} \\
& \frac{1}{2}\left[C_{1111}\left(E_{11}^{I}\right)^{2}+2 C_{1122} E_{11}^{I} E_{22}^{I I}+C_{2222}\left(E_{22}^{I I}\right)^{2}\right]=U_{I+I I}
\end{aligned}
$$

Here, $U_{A}$ is the strain energy (10) of the superelement in strain condition $A$ with boundary conditions defined by (13), and

$U_{A+B}=U_{A}+U_{B}+\frac{1}{2} \int_{V} \sigma_{i j}^{A} \varepsilon_{i j}^{B} \mathrm{~d} V+\frac{1}{2} \int_{V} \sigma_{i j}^{B} \varepsilon_{i j}^{A} \mathrm{~d} V$

is the strain energy if the boundary conditions $A$ and $B$ are combined. In this case the macrostrains are

$E_{11}^{I}=\frac{u}{a} ; \quad E_{22}^{I I}=\frac{u}{a}$.

\section{4}

\section{Numerical results}

Numerical investigations of the spherical particle composite materials have been developed for the following properties of the constituents: $E_{\mathrm{m}}=2.6 \mathrm{GPa}$, $v_{\mathrm{m}}=0.3, E_{\mathrm{m}} \leq E_{\mathrm{p}} \leq 100 E_{\mathrm{m}}, 0.1 \leq v_{\mathrm{p}} \leq 0.45$, where $E_{\mathrm{m}}, v_{\mathrm{m}}, E_{\mathrm{p}}, v_{\mathrm{p}}$ - Young's moduli and Poisson's ratios for the matrix and the particles, respectively. Alter of the volume fraction was modeled by changing the particle's radius $\phi=\frac{4}{3} \pi \frac{R^{3}}{a^{3}}$

The finite element calculations have been performed with the ANSYS 5.3 package. 3-D 20-node isoparametric brick elements providing a parabolic displacement field have been used. The elements have three translational degrees of freedom assigned to each node. The finite element mesh consisted of 279 elements and 621 elements for particle and matrix modelling respectively (see Fig. 2), resulting to 3846 d.o.f. The calculations were performed with a Silicon Graphics Workstation "SGI IRIS4D". The calculations needed a total CPU time of $50 \mathrm{sec}$.

The dependence of the composite's effective elasticity modulus on the ratio $\frac{E_{\mathrm{p}}}{E_{\mathrm{m}}}$ for small volume fraction of the particles $(R=0.5 a)$ can be seen in Fig. 3a and in Fig. 3b for high volume fraction of the particle $(R=0.9 a)$. One can see the dependence of the effective shear modulus of the composite on the shear modulus ratio particles and matrix (Fig. 4). In Fig. 4 one can see the dependence of 


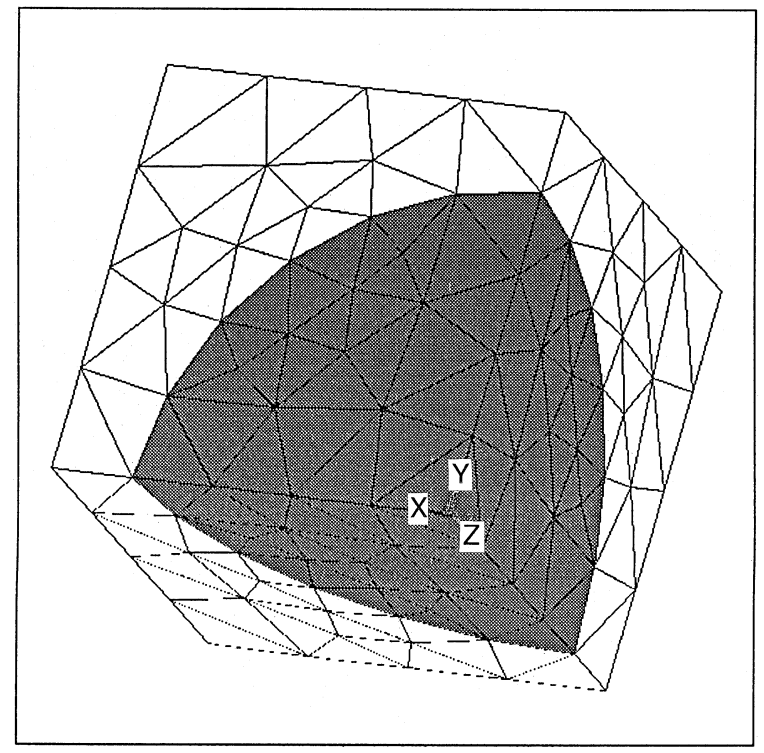

Fig. 2. Finite element discretisation of the representative volume element
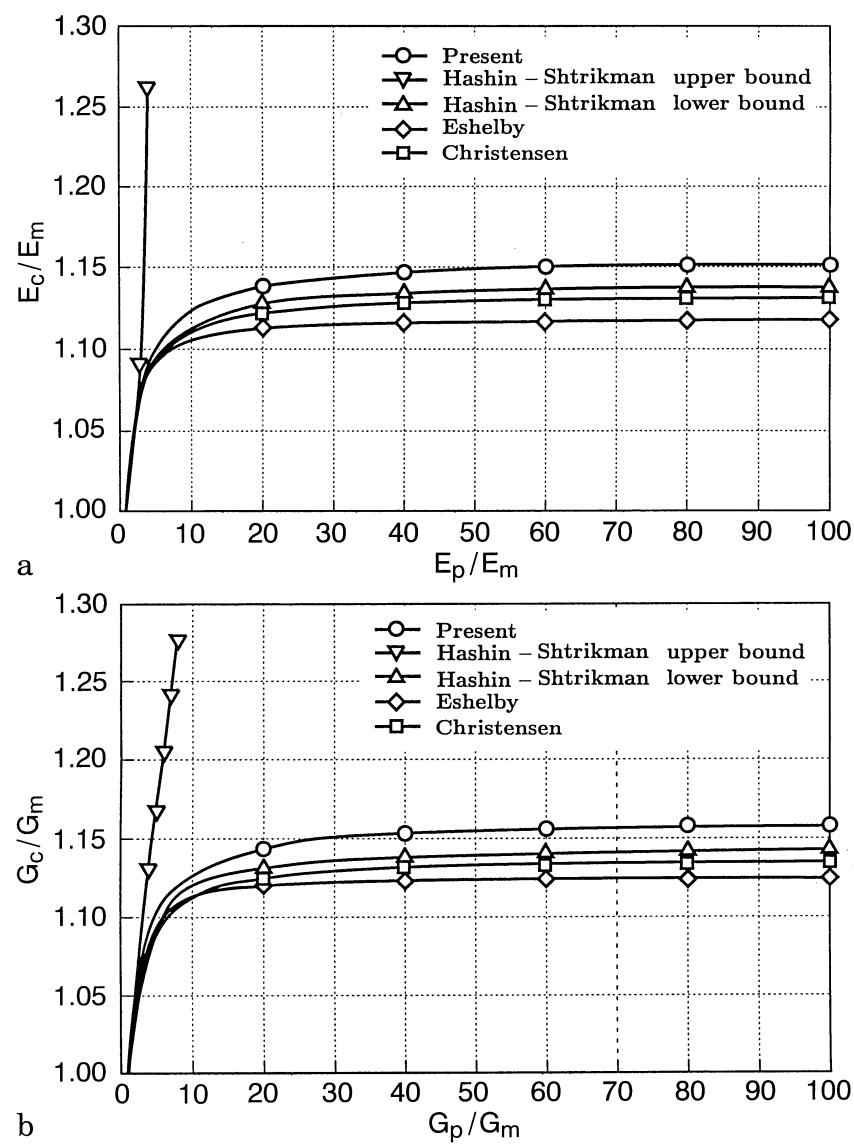

Fig. 3a, b. Dependence of effective a effective elastic and b effective shear modulus of composite with small volume fraction of the particles

the effective moduli of the composite on the volume fraction of the particles. The results of the proposed method are compared with analytical approaches discussed in Sect. 1.
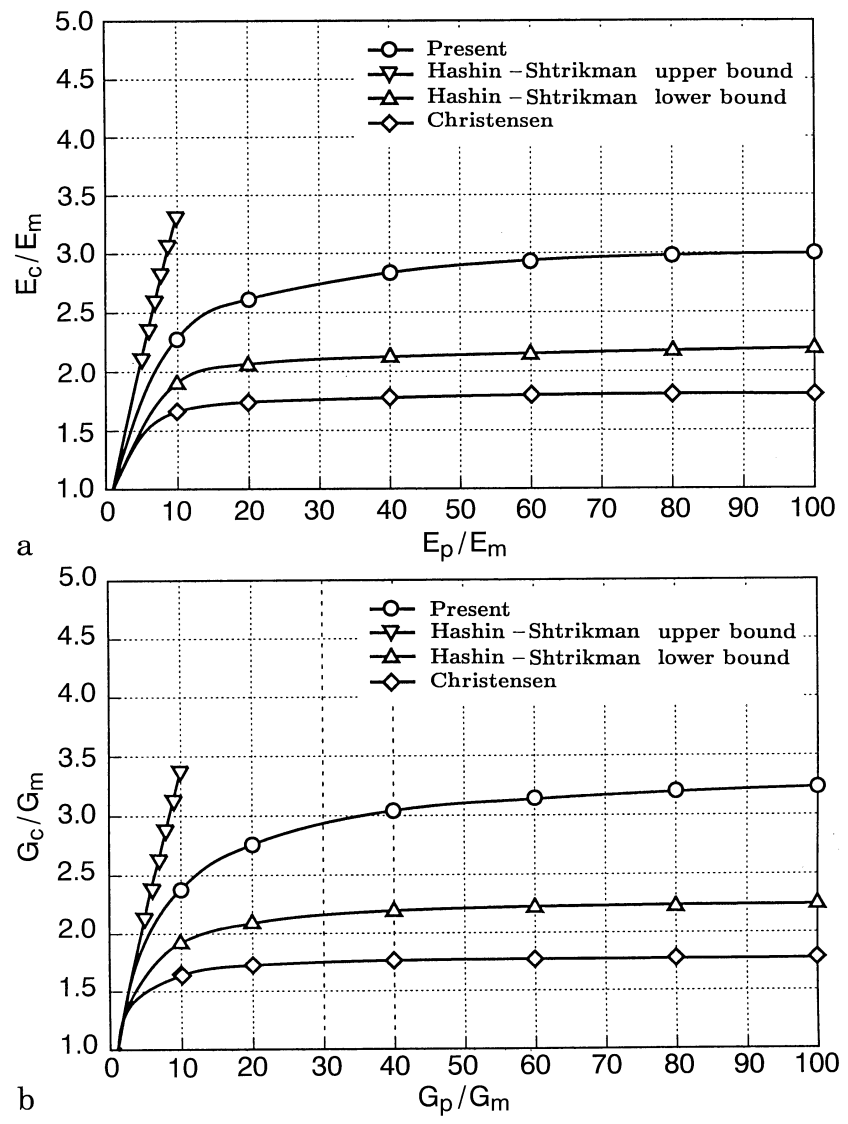

Fig. 4a, b. Dependence of effective a effective elastic and b effective shear modulus of composite with high volume fraction of the particles

\section{5}

\section{Identification procedure}

One can see that the character of the dependencies in Fig. 5 are the same for the finite element method as for the other methods. As note the bulk modulus of the composite reinforced with spherical particles has the same expression in different approaches. Moreover in Fig. 6 one can see that the dependence of the bulk modulus on the volume fraction of the particles is the same for the finite element method too. Due to this reason we have decided to investigate expression (3) for an approximation function that can be identified by a finite element solution. Rewriting the expression (3) in the following way:

$$
\frac{K_{\mathrm{c}}}{K_{\mathrm{m}}}=1+\frac{\phi\left(K_{\mathrm{p}}-K_{\mathrm{m}}\right)\left(3+4 \frac{G_{\mathrm{m}}}{K_{\mathrm{m}}}\right)}{K_{\mathrm{p}}\left(3+4 \frac{G_{\mathrm{m}}}{K_{\mathrm{p}}}\right)-3 \phi\left(K_{\mathrm{p}}-K_{\mathrm{m}}\right)}
$$

and introducing the following designations

$$
\begin{aligned}
& \zeta=\frac{K_{\mathrm{p}}-K_{\mathrm{m}}}{K_{\mathrm{p}}} \\
& \eta_{\mathrm{p}}=\frac{G_{\mathrm{m}}}{K_{\mathrm{p}}} \\
& \eta_{\mathrm{m}}=\frac{G_{\mathrm{m}}}{K_{\mathrm{m}}} \\
& \psi=\phi \zeta
\end{aligned}
$$




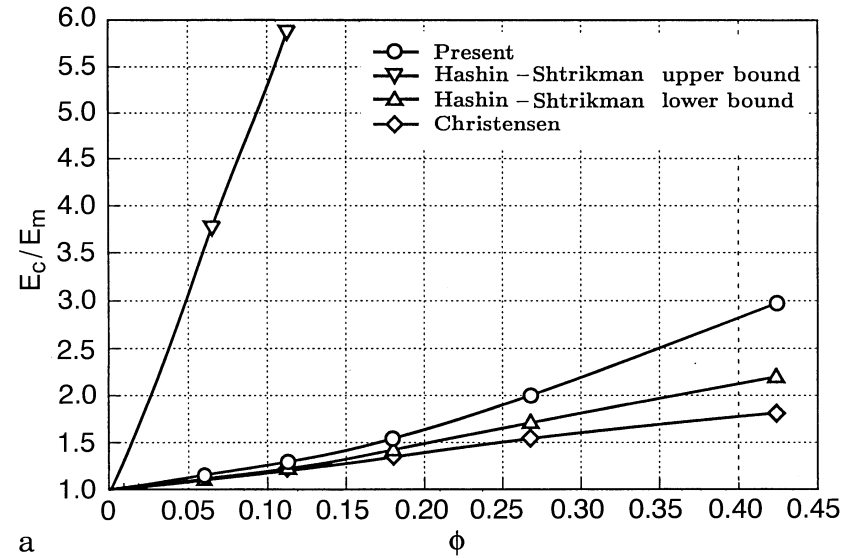

a

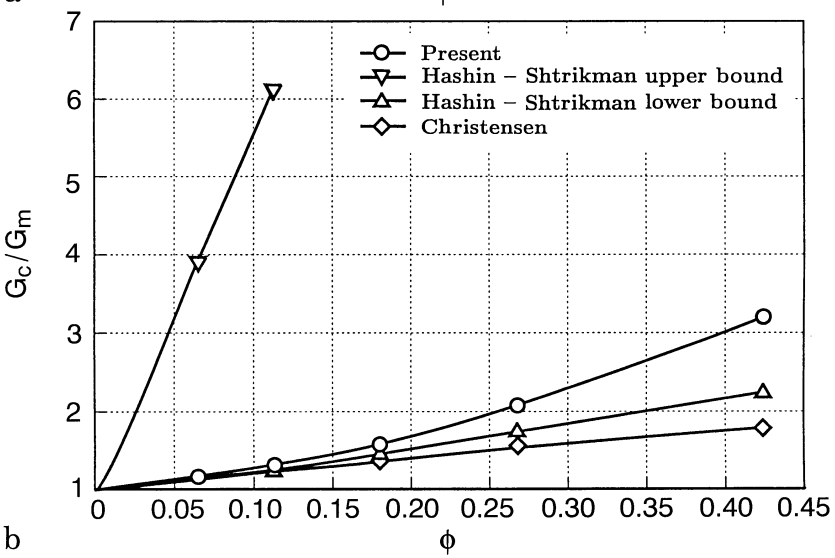

Fig. 5a, b. Dependence of the a effective elastic and $\mathbf{b}$ effective shear modulus of composite from particle volume fraction

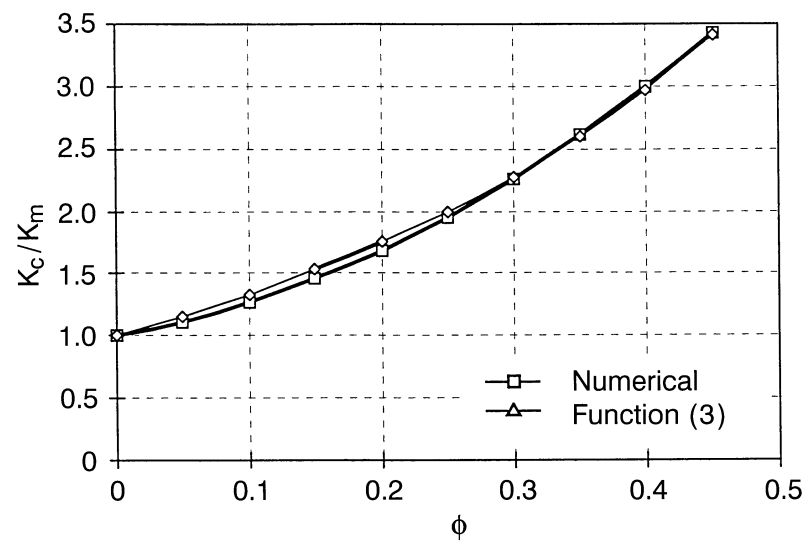

Fig. 6. Dependence of effective bulk modulus of composite from particle volume fraction

after substituting Eq. (15) into (14) one obtains:

$\frac{K_{\mathrm{c}}}{K_{\mathrm{m}}}=1+\frac{\psi\left(3+4 \eta_{\mathrm{m}}\right)}{3+4 \eta_{\mathrm{p}}-3 \psi}$

Taking into account that

$(1+\epsilon)^{-n} \simeq 1-n \epsilon \quad(|\epsilon| \ll 1)$

and supposing $\psi<<1$ the expansion of the divisor at the right part of the equation (16) leads to
$\frac{K_{\mathrm{c}}}{K_{\mathrm{m}}} \simeq 1+\psi\left(3+4 \eta_{\mathrm{m}}\right)\left(3+4 \eta_{\mathrm{p}}+3 \psi\right)$

Let us assume that the right part of Eq. (17)

$F=1+\psi\left(3+4 \eta_{\mathrm{m}}\right)\left(3+4 \eta_{\mathrm{p}}+3 \psi\right)$

is given in terms of series as

$F=1+\phi \zeta\left(a_{1}^{1}+a_{2}^{1} \eta_{\mathrm{m}}\right)+\phi^{2} \zeta^{2}\left(a_{1}^{2}+a_{2}^{2} \eta_{\mathrm{m}}\right)$

Now we can write down for the composite bulk modulus a more general expression

$\frac{K_{\mathrm{c}}}{K_{\mathrm{m}}}=1+\sum_{n=0}^{\infty}(\phi \zeta)^{n} \sum_{l=0}^{\infty} a_{l}^{n} \eta_{\mathrm{m}}^{l}$

We have assumed that the expression for elastic and shear moduli have the same from as for the bulk modulus, therefore we must only change the parameter $\zeta$ which includes corresponding elastic and shear moduli of the composite constituents

$\frac{M_{\mathrm{c}}}{M_{\mathrm{m}}}=1+\sum_{n=1}^{\infty}\left(\phi \zeta_{M}\right)^{n} \sum_{l=0}^{\infty} a_{l}^{n} \eta_{\mathrm{m}}^{l}$
$\zeta_{M}=\frac{M_{\mathrm{p}}-M_{\mathrm{m}}}{M_{\mathrm{p}}}, M=E, G, K, v$

For the determination of the effective properties of the particle composite one must identify the unknown coefficients $a_{l}^{n}$. For the reason that the finite element approach allows to calculate elastic and shear moduli, we propose to define unknown coefficients in Eq. (21) for these two expressions.

Regression analysis of the numerical results presented in Fig. 5a shows that the dependence of the effective composite elastic modulus on the particle's volume fraction is quadratic. Thus the approximation function for composite effective modulus has been searched for $n=1,2$. Here we focus our discussion on the first term of the series expression (21).

A minimization procedure of the function (21) with the numerical results (presented in Fig. 5a) leads to the following function for description of the effective elastic modulus of the composites reinforced with spherical particles:

$$
\begin{aligned}
\frac{E_{\mathrm{c}}}{E_{\mathrm{m}}}= & 1+\phi \zeta_{E}\left(1.643+0.758 \eta_{\mathrm{m}}\right)+\left(\phi \zeta_{E}\right)^{2} \\
& \times\left(5.324+2.457 \eta_{\mathrm{m}}\right), \\
\frac{G_{\mathrm{c}}}{G_{\mathrm{m}}}= & 1+\phi \zeta_{G}\left(1.613+0.744 \eta_{\mathrm{m}}\right)+\left(\phi \zeta_{G}\right)^{2} \\
& \times\left(6.408+2.957 \eta_{\mathrm{m}}\right) \\
\zeta_{E}= & \frac{E_{\mathrm{p}}-E_{\mathrm{m}}}{E_{\mathrm{p}}}, \zeta_{G}=\frac{G_{\mathrm{p}}-G_{\mathrm{m}}}{G_{\mathrm{p}}}
\end{aligned}
$$

It can be seen in Fig. 7 that the function (22) do not satisfy the numerical results for any ratio $\frac{G_{\mathrm{p}}}{G_{\mathrm{m}}}$. The reason for this is that we analyzed the dependence of the effective elastic composite modulus on the above mentioned ratio. One can see this dependence at the Fig. 3a. A regression analysis of the numerical results allows to conclude that the dependence of the effective elastic modulus of the com- 


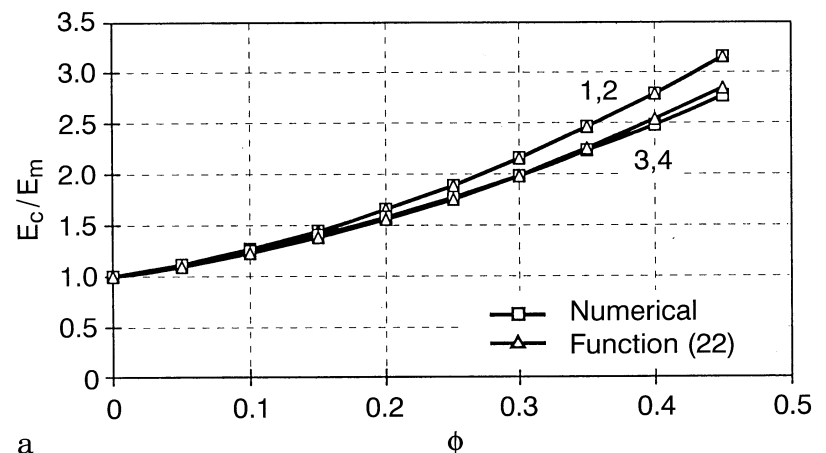

a

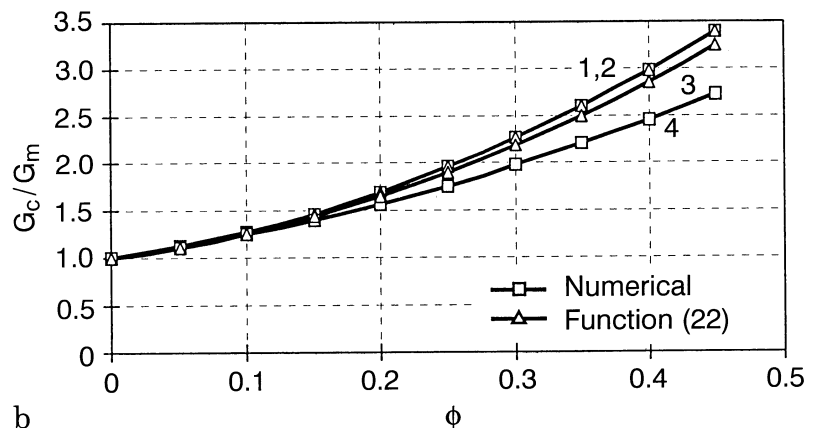

Fig. 7a, b. Comparison of a effective elastic and $\mathbf{b}$ effective shear modulus of composite from numerical investigation and approximation function (22) (Curves: 1 and 2 correspond to material with $E_{\mathrm{p}}=208 \mathrm{GPa}, v=0.3, E_{\mathrm{m}}=2.6 \mathrm{GPa}, v=0.3 ; 3$ and 4 to material with $E_{\mathrm{p}}=52 \mathrm{GPa}, v=0.4, E_{\mathrm{m}}=2.6 \mathrm{GPa}$, $v=0.3)$

posite on the ratio between the elastic moduli of particles and matrix can be described by a logarithmic function. This allows to correct the function (21)

$\frac{M_{\mathrm{c}}}{M_{\mathrm{m}}}=1+\sum_{n=1}^{\infty}\left(\phi \zeta_{M}\right)^{n}\left(\ln \left(1-\zeta_{M}\right)\right)^{n-1} \sum_{l=0}^{\infty} a_{l}^{n} \eta_{\mathrm{m}}^{l}$

The minimization of Eq. (23) leads to the following expressions for the effective elastic and shear moduli of the composite:

$$
\begin{aligned}
\frac{E_{\mathrm{c}}}{E_{\mathrm{m}}}= & 1+\phi \zeta_{E}\left(1.643+0.758 \eta_{\mathrm{m}}\right) \\
& -\left(\phi \zeta_{E}\right)^{2} \ln \left(1-\zeta_{E}\right)\left(1.215+0.561 \eta_{\mathrm{m}}\right) \\
\frac{G_{\mathrm{c}}}{G_{\mathrm{m}}}= & 1+\phi \zeta_{G}\left(1.614+0.745 \eta_{\mathrm{m}}\right) \\
& -\left(\phi \zeta_{G}\right)^{2} \ln \left(1-\zeta_{G}\right)\left(1.49+0.688 \eta_{\mathrm{m}}\right)
\end{aligned}
$$

The results presented in Fig. 8 show that the obtained functions (24) describe precisely the numerical results for any ratio of the material constituents.

\section{6}

\section{Volume expansion properties}

Since the composite material which is reinforced by spherical particles is macroisotropic, it's effective bulk modulus can be obtained from known elastic and shear moduli by a simple expression for isotropic materials:

$K=\frac{E G}{9 G-3 E}$
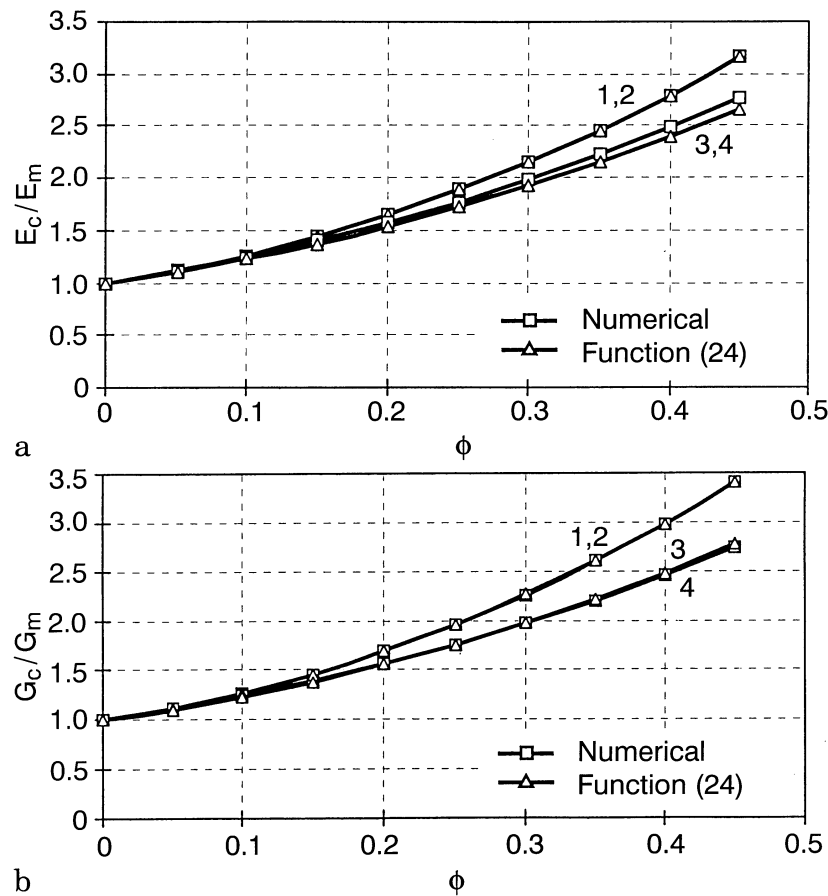

Fig. 8a, b. Comparison of a effective elastic and $\mathbf{b}$ effective shear modulus of composite from numerical investigation and approximation function (24) (Curves: 1 and 2 correspond to material with $E_{\mathrm{p}}=208 \mathrm{GPa}, v=0.3, E_{\mathrm{m}}=2.6 \mathrm{GPa}, v=0.3 ; 3$ and 4 to material with $E_{\mathrm{p}}=52 \mathrm{GPa}, v=0.4, E_{\mathrm{m}}=2.6 \mathrm{GPa}$, $v=0.3)$

In Fig. 9a one can see a good agreement between results for the composite bulk modulus obtained from the finite element approach and functions (24), (25). All effects including expansion of volume are expressed through bulk modulus of the material. Knowing the functions (24) and taking into account expression (25) one can obtain these characteristics. For the thermal expansion coefficient Rosen and Hashin (1970) have proposed:

$$
\begin{aligned}
\alpha_{\mathrm{c}}=\langle\alpha\rangle+ & \left(\alpha_{\mathrm{m}} \frac{K_{\mathrm{p}} K_{\mathrm{m}}}{K_{\mathrm{p}}-K_{\mathrm{m}}}-\alpha_{\mathrm{p}} \frac{K_{\mathrm{p}} K_{\mathrm{m}}}{K_{\mathrm{p}}-K_{\mathrm{m}}}\right) \\
& \times\left[\frac{1}{K_{\mathrm{c}}}-\left\langle\frac{1}{K}\right\rangle\right]
\end{aligned}
$$

where

$$
\begin{aligned}
\left\langle\frac{1}{K}\right\rangle & =\phi \frac{1}{K_{\mathrm{p}}}+(1-\phi) \frac{1}{K_{\mathrm{m}}} \\
\left\langle\frac{1}{\alpha}\right\rangle & =\phi \frac{1}{\alpha_{\mathrm{p}}}+(1-\phi) \frac{1}{\alpha_{\mathrm{m}}}
\end{aligned}
$$

Equation (26) can be transformed into the following form

$$
\frac{\alpha_{\mathrm{c}}}{\alpha_{\mathrm{m}}}=1+\frac{1}{1-\zeta_{\alpha}} \frac{\zeta_{\alpha}}{\zeta_{K}}\left(1-\frac{K_{\mathrm{m}}}{K_{\mathrm{c}}}\right),
$$

where

$$
\zeta_{\alpha}=\frac{\alpha_{\mathrm{p}}-\alpha_{\mathrm{m}}}{\alpha_{\mathrm{p}}}, \zeta_{K}=\frac{K_{\mathrm{p}}-K_{\mathrm{m}}}{K_{\mathrm{p}}}
$$




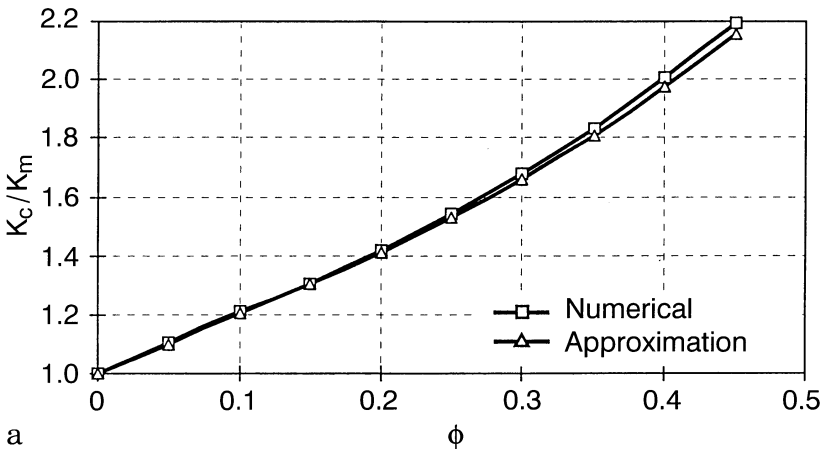

324

a

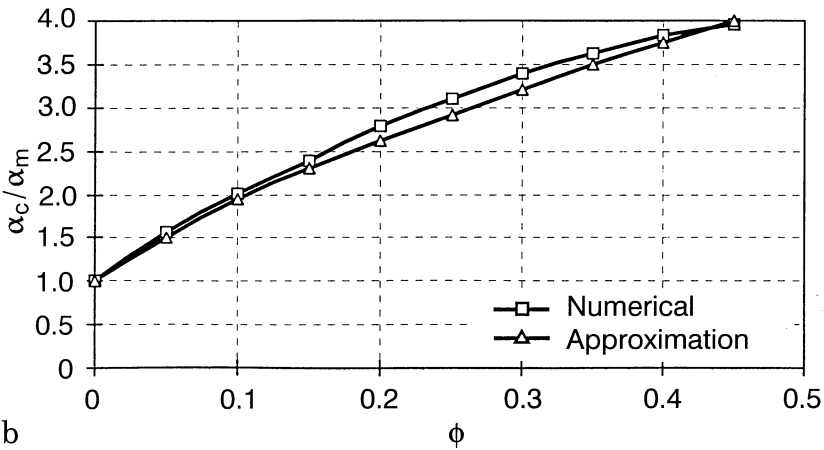

Fig. 9a, b. Effective a bulk modulus and b effective thermal expansion coefficient of composite

We have entered in expression (27) the effective bulk modulus of the composite that include approximation (24) and have compared it with results of a finite element approach proposed in Sect. 3 boundary conditions for uniform volume expansion. The results of the comparison are shown in Fig. 9b. Such approach can be used to calculate other effective properties of the composite which are dependent on the volume expansion process.

\section{7}

\section{Conclusions}

The obtained functions (24) describe precisely the numerical data for any relations between material constituents. The bulk modulus can be obtained from simple relationships for elastic constants of the isotropic material since the investigated material is macroisotropic. It allows to obtain the material properties which are connected with different volume expansion of the structure (thermal expansion coefficient, specific heat capacity, etc.). The finite element method allows the modeling of materials with plastic or creep behaviour, so further investigations can be directed to extensions of the proposed method to these cases. The coefficients in function (24) can be refined with physical tests data of the real composite.

\section{References}

Araujo AL, Mota Soares CM, Moreira De Freitas MJ (1996) Characterization of Material Parameters of Composite Plate Specimens Using Optimization and Experimental Vibration Data. Composites: Part B 27B:185-191

Bolognini L, Riccio F, Bettianli F (1993) A modal technique for the identification of stiffness and mass parameters in large structures. In: C.A. Brebbia and G.M. Carlomagno (eds) Computational Methods and Experimental Measurements VI, Vol. 2: Stress Analysis (Elsevier Applied Science, London-New York) 337-352
Budiansky B (1965) On the elastic moduli of some heterogeneous materials. J. Mech. Phys. Solids 13:223-227

Christensen RM, Lo KH (1979) Solutions for effective shear properties in three phase sphere and cylinder models. J. Mech. Phys. Solids 27:315-330

Christensen RM (1990) A critical evaluation for a class of micromechanics models. J. Mech. Phys. Solids 38:379-404

Chingshen Li, Ellyin F (1994) Short crack trapping/untrapping in particle reinforced metal-matrix composites. Comp. Sci. Technl. 52: $117-124$

Einstein A (1905) Eine Neue Bestimung der Moleküldimensionen. Annalen des Physik 19:289-306. English translation In: Investigations on the Theory of Brownian Motion, Dover, 1956, 36-62 Eshelby JD (1957) The determination of the field of an ellipsoidal inclusion and related problems. Proc. of the Royal Soc. of London A, No. 241., 376-396

Frederiksen PS (1992) Identification of Material Parameters in Anisotropic Plates - A Combined Numerical/Experimental Method. Ph. D. thesis, Technical University of Denmark, DCAMM Rep.

Frederiksen PS, Pedersen P (ed) (1993) Natural Vibrations of Free Thick Plates and Identification of Transverse Shear Moduli. in Optimal Design with Advanced Materials, Elsevier, New York, 131-148

Frederiksen PS (1997a) Numerical Studies for the Identification of Orthotropic Elastic Constants of Thick Plates. Eur. J. Mech. A/ Solids. 16:117-140

Frederiksen PS (1997b) Experimental Procedure and Results for the Identification of Elastic Constants of Thick Orthotropic Plates. J. Composite Mater. 31:360-382

Hashin Z (1962) The elastic moduli of heterogeneous materials. A survey. J. Appl. Mechanics 29:143-150

Hashin Z (1983) Analysis of composite materials. A survey. J. Appl. Mechanics 50:481-504

Hashin Z, Shtrikman S (1962) A variational approach to the theory of the elastic behaviour of polycrystals. J. Mech. Phys. Solids 10: 343-352

Hashin Z, Shtrikman S (1963) A variational approach to the theory of the elastic behaviour of multiphase materials. J. Mech. Phys. Solids 11: 127-140

Hill AA (1965) A self consistent mechanics of composite materials. J. Mech. Phys. Solids 13:213-222

Hollister SJ, Kikuchi N (1992) A comparison of homogenization and standard mechanics analyses for periodic porous composites. Computational Mechanics 10:73-95

Linzhi W, Songhe Meng, Shanyi Du (1997) The overall response of composite materials with inclusions. Int. J. Solids Structures 34: 3021-3039

Mc Laughlin R (1977) A study of the differential scheme for composite materials. Int. J. Engng. Sci. 15:237-244

Mota Soares CM, Moreira De Freitas M, Araujo AL, Pedersen P (1993) Identification of Material Properties of Composite Plate Specimens. Composite Structures 25:277-285

Norris AN (1985) A differential scheme for the effective moduli of composite. Mechanics of Materials 4:1-16

Pedersen P, Frederiksen PS (1990) Sensitivity Analysis for Identification of Material Parameters. Proc. 9th Int. Conf. On Experimental Mechanics, Copenhagen, 2:545-551

Pedersen P, Frederiksen PS (1992) Identification of Orthotropic Material Moduli by a Combined Experimental/Numerical Approach. Measurement, 10:113-118

Rammerstorfer FG, Böhm HJ (1994) Micromechanics for Macroscopic Material Description of FPRs. Engineering Mechanics of Fibre Reinforced Polymers and Composite Structures. CISM Courses and Lectures NO. 348 International Centre for Mechanical Sciences (ed: J. Hult and F.G. Rammerstorfer), pp. 9-50. Springer-Verlag Wien New York

Rikards R, Krasnikov A, Kushnevsky V (1991) A Finite Element Method of Determining the Effective Mechanical Characteristics 
of Three- Dimensional Reinforced Fiber Composites. Mechanics of Composite Materials 28:656-661

Roscoe R (1952) The viscosity of suspensions of rigidspheres.

Brit. J. Appl. Phys. 3:267-277

Rosen BW, Hashin Z (1970) Effective Thermal Expansion Coefficients and Specific Heats of Composite Materials. Int. J. Engng. Sci. 8:157-173

Sol H (1986) Identification of anisotropic plate rigidities using free vibration data. $\mathrm{PhD}$ thesis, Free University of Brussels (VUB) Sol H, De Visscher J, De Wilde WP (1993) Identification of the viscoelastic material properties of orthotropic plates using a mixed numerical/experimental technique. In: C.A. Brebbia and G.M. Carlomagno (eds): Computational Methods and Experimental Measurements VI, Vol. 2: Stress Analysis, pp. 131-142. London-New York: Elsevier Applied Science

Suquet P (1987) Elements of homogenization theory for inelastic solid mechanics. In: Sanchez-Palencia, E.; Zaoui, A. (eds): Homogenization techniques for composite media, pp. 194-278. Berlin, Heidelberg, New York: Springer

Sun QP, Hwang KC, Yu SW (1991) A micromechanics constitutive model of transformation plasticity with shear and dilatation effect. J. Mech. Phys. Solids 39:507-524 